\title{
An Intelligent Approach for Improving the Effectiveness of Smart Computing
}

\author{
Khushboo Roy \\ M.Tech $2^{\text {nd }}$ YEAR \\ Kamla Nehru Institute of Technology, Sultanpur
}

\author{
Neelendra Badal, $\mathrm{PhD}$ \\ Professor and Head of Department \\ Kamla Nehru Institute of Technology, Sultanpur
}

\begin{abstract}
Devices can communicate and share information in a smart environment using new generation technology. Smart computing, next-generation computing, is a combination of hardware, software, and network to provide real-time awareness of real-time systems. It aims to monitor, analyzing, and reporting in a faster and smarter way to make a system, as a smart system. This study follows the identification of the various factors that may affect the effectiveness of smart computing. As a result, some factors were identified hypothetically using a comprehensive literature review approach. After performing the analysis, the most important factors were identified. Then, an intelligent approach is applied for improving the effectiveness of smart computing. Lastly, the conclusion concludes along with the future scope which summarizes the work.
\end{abstract}

\section{Keywords}

Smart Computing, factors, CLS model, relationship model

\section{INTRODUCTION}

Today, a world is full of smart technology working in a smart environment with smart devices where each device can connect, communicate, and transfer information to one another[21].Computers are performing a more secure and faster computation. Examples of such smart environments consuming smart technologies are Smart-watches, smartphones smart-homes, smart-cities, smart agriculture, and so on. In this paper firstly, an introduction of smart computing is covered. The next step is the identification of affecting factors for the effectiveness of smart computing. The objective is for identifying and reviewing all the factors that affect the $5 \mathrm{~A}$ 's of smart computing. Also, the most important factors identified among them. This is done by identifying the factors hypothetically based on the model. Next, is about an improvement in the effectiveness of smart computing. The objective is to improve its effectiveness. This is done by predicting a dependent factor using independent factors using classification (categorical value prediction) and application of linear regression techniques.

Lastly, the work concludes by suggesting an intelligent approach to identify the factors and how they affect the effectiveness of smart computing along with the future scope.

\section{SMART COMPUTING}

Smart Computing is a combination of two words: Smart and Computing, where SMART means Self-Monitoring, Analyzing, and Reporting Technology. And Computing means performing computational analysis. Therefore, it collects and stores the data, monitors, and detects for what purpose it was designed, analyzes it, and report in advance accordingly to the user.

According to researchers, SMART refers to Specific
Measurable / Measurement Achievable Relevant TimeOriented and computing to perform calculations quickly. Monitoring, analyzing, and reporting in a faster and smarter way to make a system, as a smart system. The main focus of smart computing is to provide cheaper technology, solving an existing problem, and look ahead to future problems and preempt them before happening. It is a sensor-based technology, with the combination of the Internet of things (IoT), machine learning, Big Data, Artificial Intelligence, etc.

\subsection{Background}

Not since today but from the era's, computer technologies are working on its tools and techniques to make our daily lives more simple and easier[26]. Today every object is connected and communicates with each other in an environment. These objects are hardware and software devices we use in our daily lives that communicate using the internet to share their data and current status over the internet[10][20].

The technology through which the hardware and software devices communicate with each other using the internet and share information is known as SMART COMPUTING[5][9][20][26]. It is the next generation of computing that is used to create something self-aware that is it can sense the activities of its environment, massages the gathered information, perform some analytics, and provides the optimal decisions along with predicting future risks and challenges. In this, the communication process takes place among devices through

\section{-Sensors \\ -Development boards-Arduino, Raspberry-Pi -Cloud}

Sensors may be hardware or software components that sense the physical world and share the information with the development boards. This development board processes the data with the help of controllers and processors and triggers a task. And the cloud securely stores the shared information with the help of the network and transmits back when required[2]. To store the information on the cloud and for the communication process, a communicating language such as python, C, or Java and communicating protocols such as HTTP, MQTT, WebSockets are used. To make the process more secure, only the authorized person is allowed to access the data.

\subsection{Components of smart computing}

The components explain how the information travels in this technology and how smart computing functions. These are as follows:

- Sensors devices for sensing the data from hardware and software devices

- Device processors for processing and learning with the previous data 
- Cloud for storing the information

- Communicating protocols such as HTTPs or MQTT

- Communicating language such as python In smart computing, there is $70 \%$ of the hardware utilization that is more use of resources with the smart OS. Smart OS makes computation faster, efficient, and smarter.

\section{3 $5 \mathrm{~A}$ 's of smart computing}

Smart computing comprises five key functions which are 5 A's of smart computing[5][10][26] that is discussed below:
a. Awareness
b. Analysis
c. Alternative
d. Action
e. Auditability

All the functionality of smart computing evolves around these 5 A's and analyzing, reporting and monitoring functions are performed accordingly. The detailed discussion of the 5 A's are discussed below in Table1:

Awareness is the most important A among all the 5 A's of smart computing. Innovations in Awareness will have the most revolutionary impact, the statement justifies as if there is a lack of awareness, then the analytical tool detects and stores the wrong information. An alternative triggers the wrong action that makes a wrong decision regarding which may lead to a serious problem in the future.

Table 1. Description of $5 \mathrm{~A}$ 's of smart computing

\begin{tabular}{|c|c|c|}
\hline S.No & 5 A's & Description \\
\hline A & Awareness & $\begin{array}{l}\text { Identification of tools and } \\
\text { devices, use of sensors, GPS, } \\
\text { RFID[22] } \\
\text { Identification of customers(a } \\
\text { type of user, identity, location, } \\
\text { status)[5][26][28] } \\
\text { Identification of integrated } \\
\text { communication technology such } \\
\text { as } 3 \mathrm{G}, 4 \mathrm{G} \text {, etc.[5] }\end{array}$ \\
\hline B & Analysis & $\begin{array}{l}\text { - } \begin{array}{l}\text { Easy and adaptable servers } \\
\text { - Storage device enabled by server } \\
\text { visualization }\end{array} \\
\text { - } \quad \text { Data-center automation } \\
\text { - Strage life cycle management } \\
\text { - Increased storage capacity }\end{array}$ \\
\hline $\mathrm{C}$ & Alternatives & \begin{tabular}{|l} 
Identify rule engines and \\
workflow[31] \\
What decision should trigger at \\
necessary actions
\end{tabular} \\
\hline $\mathrm{D}$ & Action & $\begin{array}{l}\text { Correct action occurs at the } \\
\text { correct time } \\
\text { On-time notification } \\
\text { Type of process application } \\
\text { (action will execute through } \\
\text { integrated links to } \\
\text { application)[10] }\end{array}$ \\
\hline
\end{tabular}

\begin{tabular}{|c|l|l|}
\hline $\mathrm{E}$ & \multirow{2}{*}{ Auditability } & $\begin{array}{l}\text { Technology needs to capture, } \\
\text { track and analyze information } \\
\text { What actions are taken(right or } \\
\text { wrong) } \\
\end{array}$ \\
& & $\begin{array}{l}\text { How to improve analysis } \\
\text { Identify } \\
\text { alternatives[5][10]. }\end{array}$ \\
\hline
\end{tabular}

For example, the train tracking system tracks and navigate the exact position of the train. This tracking system provides information about the free track for the trains. If there is any train on one track train has to divert to the next track to prevent the accident. However, if there is a lack of awareness, the system transmits wrong information about the track and predicts there is no obstacle on the path. This wrong information is passed further for analytics and the data about the blank track which is not blank is stored and accordingly, the wrong action is taken which may lead to long waiting or take a wrong path or may lead to an accident. Hence, proper awareness and improved analytical tools to be used to avoid such problems. There must be proper fine-tuning among 5A's of smart computing[5].

\section{REVIEW RELATED TO SMART COMPUTING}

In Forrester's white paper report, Andrew H. Bartels [5] gives a basic idea of smart computing along with its $5 \mathrm{~A}$ 's that are its key functions and discusses how can help in driving the new era of IT growth. This report explains the evolutions in the generation of computing. The main focus is to explain how smart computing helps in predicting future business risks and the growth of the market in the field of technology.

Mrs. Monika Garg, Mr.Promod Kumar, Miss Swati Aggrawal[27] explains how green computing is smart computing. The idea behind is energy efficient energy and eco-friendly computing. Effective coding is to conserve resources to make less use of the hardware by having the program.

Matthew N. O. Sadiku, Yu Zhou, and Sarhan M. Musa [26] provide a detailed review of the introduction of smart computing and its application areas.

Muntasser A.Wahsh and Jaspaljeet Singh Dhillion [25] reviews the factors that affect the adoption of cloud computing for e-government and public sectors. This study follows a systematic literature review (SLR) approach that helps to identify what are the various factors that affect cloud computing. As a result, Fifty-nine factors were identified based on their frequencies and seven among them appear to be most important.

Apurva Adapa, RichardH.Hall, Samuel N.Smith, Fiona FuiHoon Nah, and Keng Siau [6] explores the contributing factors and their relationships that influence the adoption of wearable devices through in-depth interviews using a ladder approach. This research also provides information about using the laddering approach to data collection and analysis.

Luis AugustoSilva, Valderi Reis Quietinho Leithardt, Carlos O Rolim, Gabriel Villarrubia Gonzalez, Claudio F.R.Geyer, and Jorge Sa Silva [11] develops an alert and a notification management system. The study discussed how the number of users and devices is increasing daily and their tolerance may decrease in a short time. Hence there is a need to develop a system.

Adela Jukan, Xavi Masip-Bruin, and Nina Amla [2] discusses 
applications of smart computing. It provides the application of smart computing sensing technologies for domestic, farm, and wild animal welfare.

Tae-Gyu Lee, Seong-Hoon Lee, and Gi-Soo Chung [23] discusses the structural and functional issues of smart computing and how smart computing interact with cloud computing for contributing accessible information. Two proposed work: Smart table and Smart control algorithm. Robert E.Kahn [29] highlights the generations of computing. The study provides a comparative study of five generations of computers and computing technologies based on computer hardware, computer software, telecommunication technologies, and computer performance. What qualities do an intelligent system must possess and advancements in different fields of computing technologies are also considered.

\section{THE IDENTIFICATION OF AFFECTING FACTORS FOR THE EFFECTIVENESS OF SMART COMPUTING}

The key factors of smart computing and are closely connected and dependent on one another. At very first, the awareness is performed which means to get aware of what could happen next by collecting real-point data with the help of sensors, RFIDs, or any devices that capture the information of the customers, or identify the location, or check the current status[20]. This awareness is dependent on some analysis, which is done using some business intelligence and analytical tools to check whether this alert is important or can be ignored. Thirdly, identify the alternative action that can be done in response to anomaly using the rule engines and workflow. And accordingly, actions are performed. Lastly, capturing and analyzing the data on activity at every stage for future problems and improvements using auditability. All the 5 A's, anyhow, may depend on some factors which affect them. Factors of smart computing are identified hypothetically by the following questions

- Which factors influence the 5 A's of smart computing?

- Which factors are more important?

- How these factors do affect the adoption of smart computing?

- What are the different challenges associated with the affecting factors?

To answer these questions, a CLS model (Comprehensive Literature Survey) is designed. This shows how previous work is beneficial in this work to identified factors based on the survey.

The following factors are identified that are associated with smart computing using the CLS MODEL.

Adaptability[1][25], Verification[2][28], Authentication[28], Authorization[28], Accessibility[2], Availability[22], Security[2], Autonomy, Flexibility, Adaptability, Reliability, Manageability, Predictability,

Fault-tolerance, Failure management, Accuracy, Maintainability, Transparency, Robustness, Reusability and so on. Among the numerous factors, Accuracy is the most important factor. 


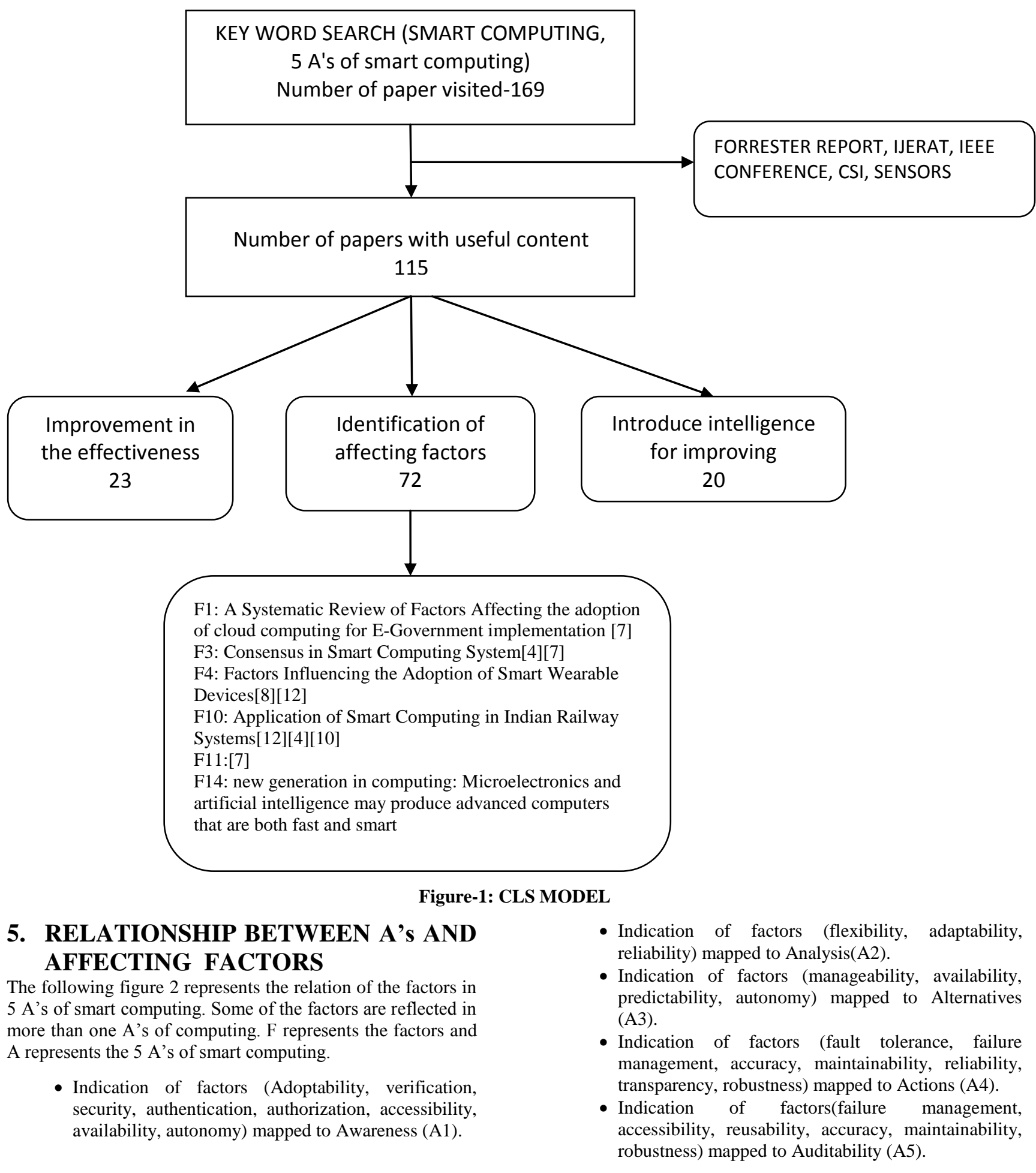




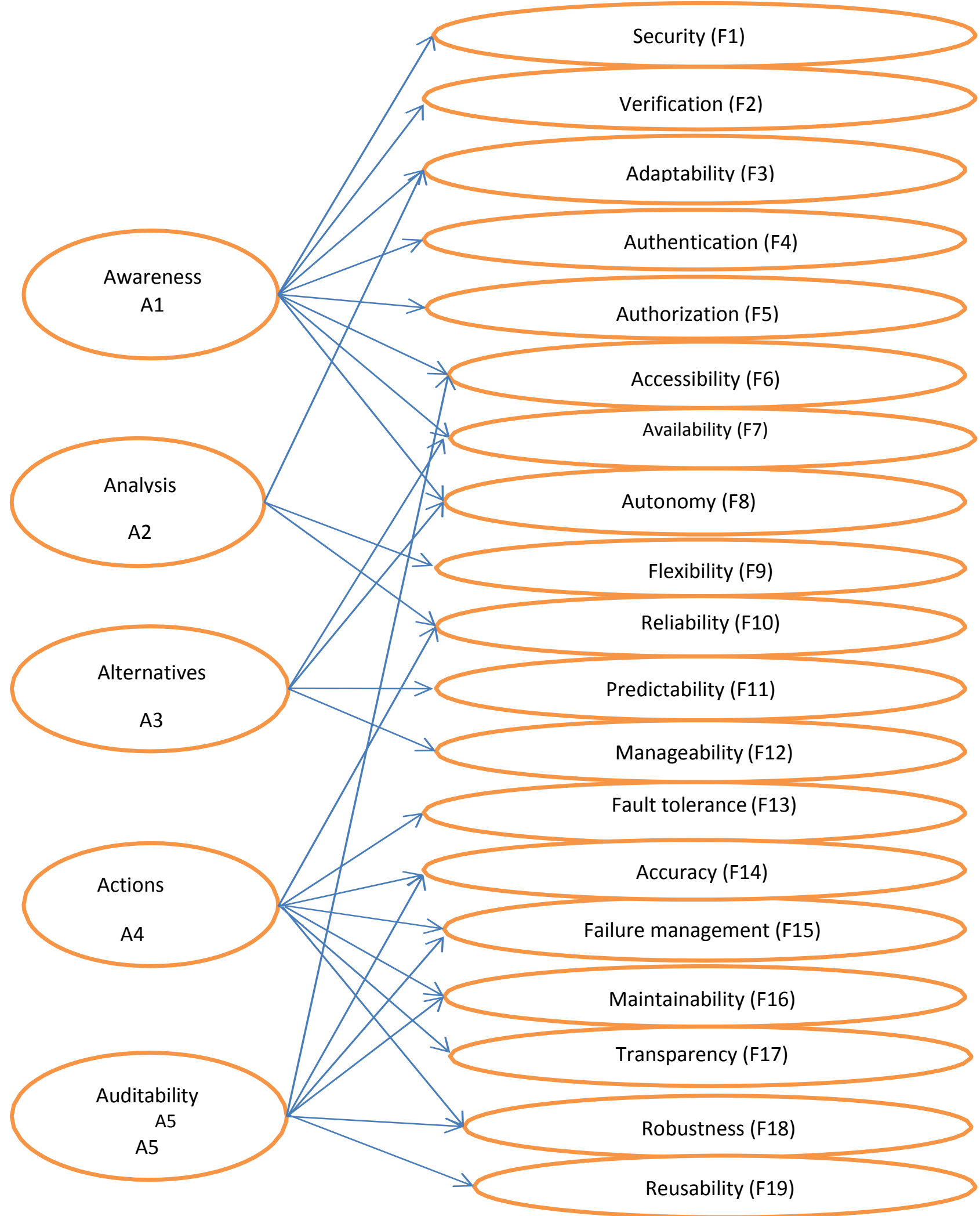

Fig 1:Relationship between factors and $5 \mathrm{~A}$ 's

6. A TECHNIQUE TO INTRODUCE INTELLIGENCY FOR IMPROVING THE EFFECTIVENESS OF SMART COMPUTING

Use of statistics to identify the relationship between the variables. This includes introducing intelligence for improving the effectiveness of smart computing correlation and regression model for identifying the factors and $5 \mathrm{~A}$ 's relationship. 


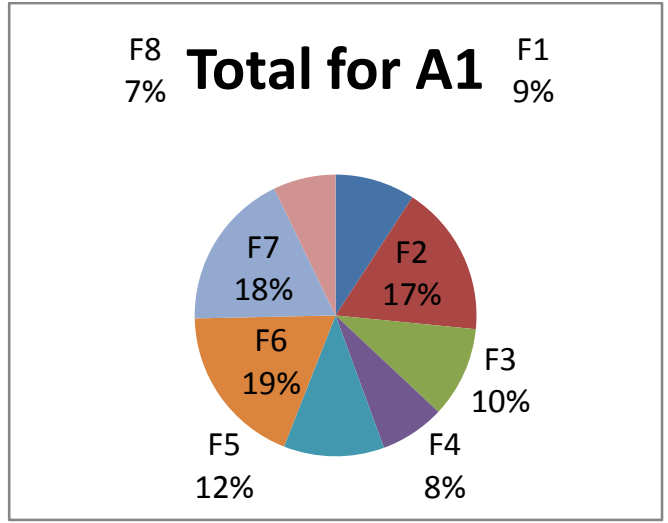

The pie-chart represents the percentage of factors against A1. The diagram shows the contribution of factors for the formation of A1.Therefore,F1 contributes 9\%,F2 contributes $17 \%, \mathrm{~F} 3$ contributes $10 \%, \mathrm{~F} 4$ contributes $8 \%, \mathrm{~F} 5$ contributes $12 \%$, F6 contributes $19 \%$, F7 contributes $18 \%$ and F8 contributes $18 \%$ for the formation of A1.

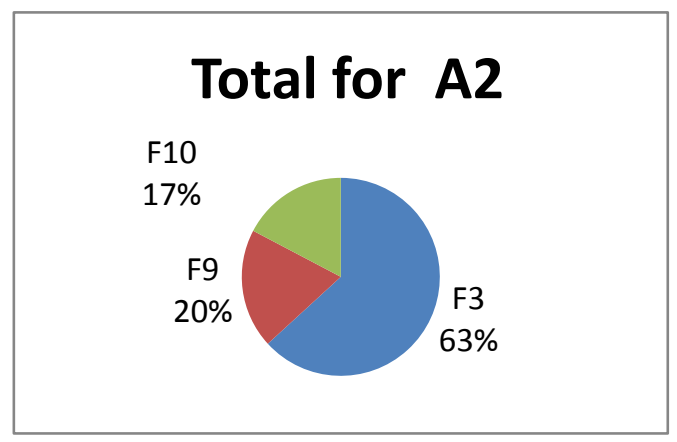

The above pie-chart represents the percentage of factors against A2. The diagram shows the contribution of factors for the formation of A2.Therefore,F3 contributes 63\%,F9 contributes $20 \%$, and F10 contributes $17 \%$.

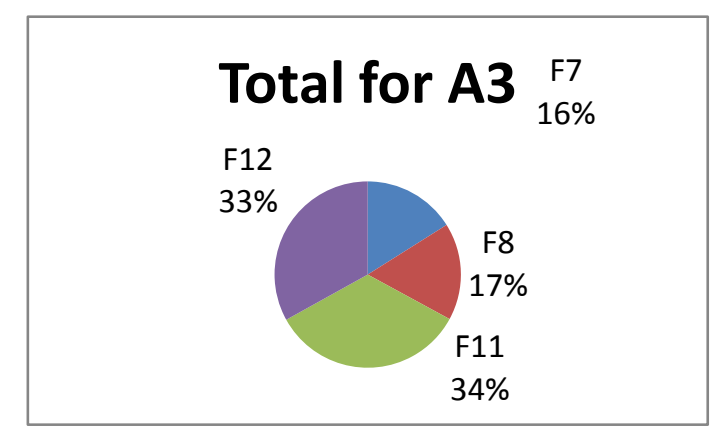

The pie-chart represents the percentage of factors against A3. The diagram shows the contribution of factors for the formation of A3.Therefore, F7 contributes 16\%, F8 contributes $17 \%, \mathrm{~F} 11$ contributes $34 \%$, and F12 contributes $33 \%$.

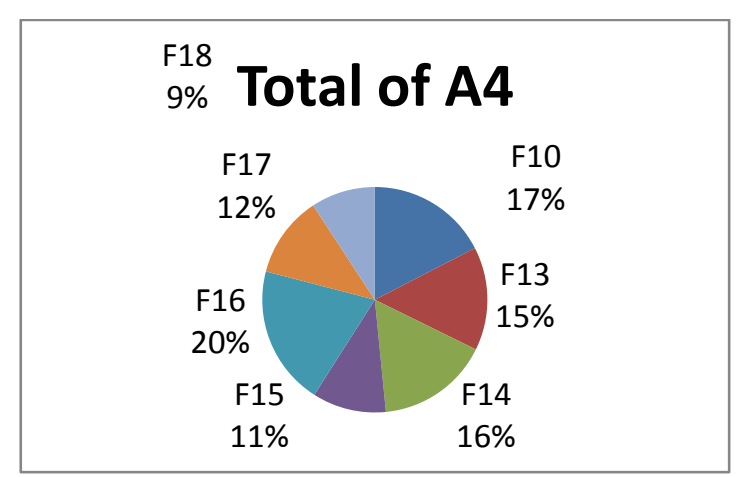

The above pie-chart represents the percentage of factors against A4. The diagram shows the contribution of factors for the formation of A4.Therefore, F10 contributes 17\%, F13 contributes $15 \%$, F14 contributes $16 \%$, F15 contributes $11 \%$, F16 contributes 20\%, F17 contributes $12 \%$ and F18 contributes $8 \%$.

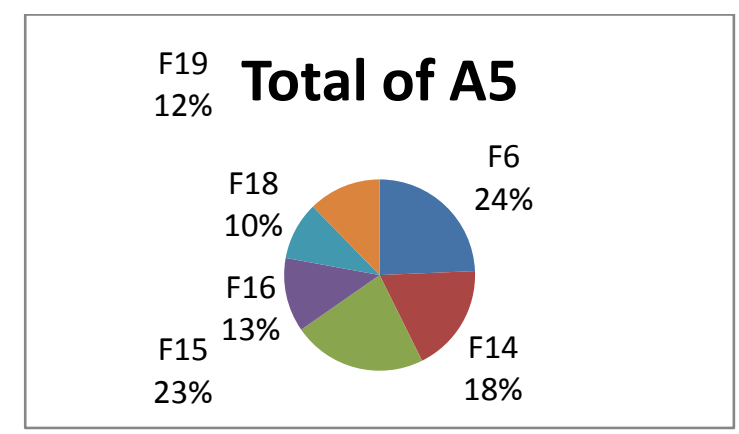

The above pie-chart represents the percentage of factors against A5. The diagram shows the contribution of factors for the formation of A5.Therefore, F6 contributes 24\%,F14 contributes $18 \%$, F15 contributes $23 \%$, F16 contributes $13 \%$, F17 contributes $12 \%, \mathrm{~F} 18$ contributes $8 \%$ and F19 contributes $12 \%$.

\section{CONCLUSION}

With the comprehensive and systematic literature survey of previous work, the factors that may affect the effectiveness of smart computing are identified. Hence, overall the work is dedicated to "The identification of affecting factors for the effectiveness of smart computing". There is also a need to analyze how these factors may affect the effectiveness of smart computing. A various literature survey was performed to identify the analyzing techniques used for smart computing. Hence, overall the work is dedicated to "An improvement in the effectiveness of smart computing".Various approaches can be used so that they can improve the effectiveness of smart computing. Hence, overall the work is dedicated to "Introduce intelligence for improving the effectiveness of smart computing".

After providing the method that improves the effectiveness of smart computing by applying an intelligent approach, the overall work is dedicated to" An Intelligent Approach for Improving the Effectiveness of Smart computing".

\section{FUTURE SCOPE}

The future scope of the work is that various other factors other than these 19 factors can be identified that can affect the effectiveness of smart computing.

The quantitative data which has been used for the analysis can have a varied and the observation can be carried with a 
different set of values

Other attributes that can affect the factors can be identified that can directly or indirectly affect the effectiveness of smart computing.

\section{ACKNOWLEDGMENTS}

I am thankful to my dissertation advisor Dr. Neelendra Badal (Professor and Head of Department, Department of Computer Science and Engineering) Kamla Nehru Institute of Technology, Sultanpur, for his valuable guidance, encouragement, and co-operation during this entire thesis work. It was his able guidance and support, which resulted in the successful work within the specified time.

I would like to thank all the people who were involved in the validation survey for this research project. Without their passionate participation and input, the validation survey could not have been successfully conducted.

Finally, I must express my very profound gratitude to my parents for providing me with unfailing support and continuous encouragement throughout my years of study and through the process of researching and writing this dissertation. This accomplishment would not have been possible without them. Thank you, last but not least, the cooperation and help received from teachers and friends of the Department of CSE, is gratefully acknowledged.

\section{REFERENCES}

[1] A.Bennani, M.Elmahouti, M.L.Kerkeb, S.Khoulji, and S.Bourekkadi, "Smart Computing Solution For Settling A Problem Of Cloud Computing Adoption Acknowledgement”, April 2018, IJSER International Journal of Scientific and Engineering Research, pp. 633640, ISSN 2229-5518

[2] Adela Jukan, Nina Amla, and Xavi Masip-Bruin, "Smart Computing and Sensing Technology for Animal Welfare: A Systematic Review", April 2017, ACM Computing Surveys, pp. 1-27, DOI: http://dx.doi.org/10.1145/3041960

[3] Akshith Kumar, Bhagyashree, and Saritha Crasta, "Tourism Opportunities in Mangalore as a Smart City", 11 May 2017, International Journal of Computational Research and Development, pp.96-101, DOI: 10.5281/zenodo. 574255

[4] Alphonse Sebastian, K. Prathapchandran, and S.Sivagurunathan, "Internet of Things for Developing Smart Sustainable Cities (SSC): A Security Perspective", 23 July 2016, DOI: 10.1007/978-3-319-33124-9_13

[5] Andrew H. Bartels, "Smart computing drives the new era of IT growth", 4 December 2009, Forrester report

[6] Apurva Adapa, Fiona Fui-Hoon Nah, Keng Siau, RichardH.Hall, and Samuel N.Smith, "Factors Influencing the Adoption of Smart Wearable Devices", 2018, International Journal of Human-Computer Interaction, 409, DOI: $10.1080 / 10447318.2017 .1357902$ pp. 399-

[7] Ashoke Nath and Parag Chatterjee, "Application of Smart Computing in Indian Railway Systems", June 2014, IJSRMS International Journal of Scientific Research and Management Studies, pp. 148-155

[8] Arun Cyril Jose and Reza Malekian, "Smart Home Automation Security: A Literature Review", 31 August
2015, The Smart Computing Review, pp. 269-285, DOI: 10.6029/smartcr.2015.04.004

[9] Athula Ginige and Janagan Sivagnanasundaram, "Enhancing Agricultural Sustainability through Crowdsensing: A Smart Computing Approach", 3 September 2019, Journal of Advanced Agricultural Technologies, pp. 161-165, DOI: 10.18178/joaaat.6.3.161-165

[10] Bob.Mcllvride, "Smart computing in real-time", January 2013, Blog

[11] Carlos O Rolim, Claudio F.R.Geyer, Gabriel Villarrubia Gonzalez, Jorge Sa Silva, Luis AugustoSilva, and Valderi Reis Quietinho Leithardt, "PRISER: Managing Notification in Multiple Devices with Data Privacy Support",13 July 2019, Sensors, pp. 1-18, DOI: $10.3390 / \mathrm{s} 19143098$

[12] Changhyun Byun, Jekuk Yun, Juno Chang, Kyugeun Park, and Yangon Kim, "Context-Aware Inference (CAI) Model on Smart Computing Environment", 2012, International Conference on Information Science and Applications, Suwon, pp. 1-6, DOI: 10.1109/ICISA.2012.6220966.

[13] Chris Testa, Jim Blake, and Lisa Washburn, "Outsourced Smart Grid Services: A Smart Approach for AMI and Beyond", July 2013, White paper Landis-and-Gyr, pp. 15 ,

[14] Christian Bremser, Gunther Piller, and Markus Helfert,“ Technology Adoption in Smart City Initiatives: Starting Points and Influence Factors", 01 January 2019, pp.7079, DOI:10.5220/0007702700700079

[15] Dan Trombley, Maggie Molina, and Neal Elliott, "A Defining Framework for Intelligent Efficiency", June 2012, ACEEE The Association of Computer Electronics and Electrical Engineers, pp. 1-53

[16] David Romero, Muztoba Ahmad Khan, Sameer Mittal, Thorsten Wuest, "Smart Manufacturing: Characteristics, Technologies and Enabling Factors", 1 January 2019, Proceedings of the Institution of Mechanical Engineers Part B Journal of Engineering Manufacture, pp. 13421361, Vol. 233, DOI:10.1177/0954405417736547

[17] Debajyoti Mukhopadhyay and Sukanta Sinha, "Smart Computing Review Smart Metric Approach to Reduce Web Service Latency Time”, October 2015, pp. 417-424, DOI: 10.6029/smartcr.2015.05.006

[18] Dionisis Kandris, Fotios Zantalis, Grigorios Koulouras, Sotiris Karabestsos, "A Review of Machine Learning and IoT in Smart Transportation", 10 April 2019, Future Internet, pp. 1-23, DOI:10.3390/fi11040094

[19] Dr M. Balamurugan, Vijaykumar S, and Sarvanakumar S.G, "Unique Sense: Smart Computing Prototype", 2015, Elsevier Procedia Computer Science 50, pp. 223-228, DOI: 10.1016/i.procs.2015.04.056

[20] D.V.Kurmude, S.N.Kakarwal, and R.R.Deshmukh, "Smart Computing: An Integrated Approach of Computing ”, March 2019, CSI Communications, pp. 1011 ,

[21] Erol Tutumluer, Hai Huang, Shihui Shen, Shushu Liu, Tong Qiu, and Yu Qian "Simulations of large-scale triaxial shear tests on ballast aggregates using sensing 
mechanism and real-time (SMART) computing", Elsevier, 2019, pp. 184-198, DOI: 10.1016/j.compgeo.2019.02.010

[22] F. O. Akgul and K. Pahlavan, "Location awareness for everyday smart computing", 2009 International Conference on Telecommunications, Marrakech, 2009, pp. 2-7, DOI: 10.1109/ICTEL.2009.5158609.

[23] Gi-Soo Chung, Seong-Hoon Lee and Tae-Gyu Lee, "An Interaction System Architecture and Design between Smart Computing and Cloud Computing", 2012, Springer-Dordrecht, pp. 197-205, DOI: 10.1007/978-94007-5699-1 21

[24] Huaguan Li, Weigang Wu, Yu Zhou, and Zhiwei Yang, "Consensus in Smart Computing System", 2017, IEEE International Conference on Smart Computing (SMARTCOMP), Hong Kong, 2017, pp. 1-8, DOI: 10.1109/SMARTCOMP.2017.7946994.

[25] Jaspaljeet Singh Dhillon and Muntasser A.Wahsh, "A Systematic Review of Factors Affecting the adoption of cloud computing for E-Government implementation", 23 December 2015, ARPN Journal of Engineering and Applied Sciences, Volume-10, pp. 17824-32.

[26] Matthew N. O. Sadiku, Sarhan M. Musa, and Yu Zhou, "Smart Computing", July 2019, IJERAT International Journal of Engineering Research and Advanced 10.31695/IJERAT.2019.3458
[27] Mrs Monika Garg, Mr Pramod Kumar, And Miss Swati Aggrawal,“ Green Computing is SMART COMPUTING-A Survey ", February 2012, International Journal Of Emerging Technology And Advanced Engineering, pp. 297-303, Volume 2, ISSN 2250-2459

[28] Patrick George, "User Authentication With Smart Cards In Trusted Computing Architecture", 2004, Security and Management, pp. 1-7

[29] Robert. E. Kahn, "A new generation in computing: Microelectronics and artificial intelligence may produce advanced computers that are both fast and smart", November 1983, IEEE Spectrum, pp. 36-41, Volume20, DOI: 10.1109/MSPEC.1983.6370017

[30] Satyadharma Bharti and Satya Prakash Dubey, "Application of smart computing techniques in cost optimization of $1200 \mathrm{KV}$ autotransformer", 27 November 2017, Journal of Information and Optimization Sciences, pp. 1-15, ISSN 0252-2667, DOI:10.1080/02522667.2017.1374727

[31] S.Sahana, "Smart Computing- An Overview", March 2019, CSI COMMUNICATIONS, pp. 12-13, Volume42, ISSN 0970-647X.

[32] Soumadip Sen and Sourav Samanta, "Application of Smart Computing in Digital Business and e-commerce through Business Intelligence", March 2019, CSI COMMUNICATIONS, pp. 21-24, Volume-42, ISSN 0970-647X. 